\title{
The copy number and integration site analysis of IGF-1 transgenic goat
}

\author{
JIAN LIN, QIANG ZHANG, LI Q.ZHU, QING H. YU and QIAN YANG \\ Key Laboratory of Animal Physiology and Biochemistry, Ministry of Agriculture, \\ Nanjing Agricultural University, Nanjing, Jiangsu 210095, P.R. China
}

Received February 2, 2014; Accepted June 12, 2014

DOI: $10.3892 / \mathrm{ijmm} .2014 .1841$

\begin{abstract}
Transgenic animals have been used previously to study gene function, produce important proteins, and generate models for the study of human diseases. As the number of transgenic species increases, reliable detection and molecular characterization of integration sites and copy number are crucial for confirming transgene expression and genetic stability, as well as for safety evaluation and to meet commercial demands. In this study, we generated four transgenic goats by somatic cell nuclear transfer (SCNT). After birth, the cloned goat contained transferred insulin-like growth factor I (IGF-1) gene was initially confirmed using a polymerase chain reaction (PCR)-based method. The four cloned goats were identified as IGF-1 transgenic goats by southern blotting. The number of copies of the $I G F-1$ gene in each of the transgenic goats was determined. Additionally, four integration sites of the transgene in the transgenic goats with a modified thermal asymmetric interlaced (TAIL)-PCR method were identified. The four different integration sites were located on chromosomes 2, 11, 16 and 18. The present study identified the copy number and integration sites using quantitative PCR (qPCR) and TAIL-PCR, enabling the biosafety evaluation of the transgenic goats.
\end{abstract}

\section{Introduction}

Transgenic animals are a powerful tool used in the study of the function and regulation of genes in vivo, the production of important pharmaceutical proteins, and the creation of pathologic models for human disease therapy (1-3). When new transgenic animals are generated, one essential step is to identify the transgenic livestock. Novel approaches to improve the molecular characterization of transgenic livestock would have considerable

Correspondence to: Professor Qian Yang,Key Laboratory of Animal Physiology and Biochemistry, Ministry of Agriculture, Nanjing Agricultural University, 1 Wei gang, Nanjing, Jiangsu 210095, P.R. China

E-mail: zxbyq@njau.edu.cn

Key words: transgenic goat, copy number, quantitative polymerase chain reaction, integration site, thermal asymmetric interlaced-PCR economic and commercial benefits. Commonly used transgenic techniques such as somatic cell nuclear transfer always result in the random integration of multiple copies of target genes in the host genome $(4,5)$. Thus detecting the existence and expression of target genes in transgenic animals, as well as determining the copy number and insertion site in transgenic animals is crucial, as these factors can greatly affect the expression level and genetic stability of targeted genes (6-8). The random insertion of multiple copies may have marked effects, such as the inactivation of an endogenous gene following transgene insertion. Different levels of transgene expression and even silencing of the transgene may result when inserted into a heterochromatic region due to chromosome position effects $(9,10)$.

Based on the difference between the transgenic and non-transgenic animal at the DNA level (target gene-, promoter-, marker gene- and construct-specific), a number of DNA-based methods have been utilized for transgene detection, such as polymerase chain reaction (PCR), quantitative PCR (qPCR) $(11,12)$ and thermal asymmetric interlaced (TAIL)-PCR (13-15). The PCR-based detection strategies have become the core method due to their high specificity, efficiency and sensitivity (13). The number of transgene copies has traditionally been estimated by southern blotting techniques, which are tedious and timeconsuming methods and require a large amount of DNA sample for each assay. Moreover, quantification using those methods is not accurate (when multiple copies of transgenes insert into one or more loci) and yield ambiguous results (16). The emergence of the sensitivity and accuracy of qPCR technology allows for the copy numbers in transgene animals can easily be determined $(7,11)$. qPCR technology has been applied to analyze the copy number of transgenic mice (17-19), swine (7) and livestock (5).

Chromosome walking and flanking sequence cloning procedures were employed to detect the integration site of the target gene. The potential of inserting mutation renders the identification of the transgene location critical (20). Currently, a number of PCR-based methods are available for these purposes, including inverse PCR (I-PCR) (21), ligation-mediated PCR (22) and event-specific PCR (23). However, each of these methods has drawbacks when considered for wide use in transgenic animal analysis (24). Recently, improved methodologies have been developed to address these issues. The TAIL-PCR is the most successful method currently used to precisely identify transgene flanking sequences. Additionally, 
TAIL-PCR has been widely used in identifying the insert sites of transgenic animals (20). To obtain precise flanking fragments rapidly, we have developed a novel efficient method that combined the restriction method from southern blotting with TAIL-PCR procedures to analyze the integration sites.

In this study, we generated the transgenic-cloned goat that specifically expresses insulin-like growth factor I (IGF-1) in milk (25). To stimulate the molecular analysis of cloned goats, we initially used PCR and southern blotting to identify the cloned goats as IGF-1 transgenic goats. The exact integration site and copy numbers of the four individual IGF-1 transgenic goats were detected by qPCR and TAIL-PCR methods.

\section{Materials and methods}

Production of IGF-1 transgenic goats. Transgenic goats were generated by somatic cell nuclear transfer (SCNT) with $S a l \mathrm{I} / P v u \mathrm{I}$-linearized plasmid $\mathrm{pIN}$, which contained $6.1 \mathrm{~kb}$ of the goat $\beta$-casein proximal promoter region, the coding region of goat $I G F-1$ gene and $2.3 \mathrm{~kb}$ of the $3^{\prime} \beta$-casein region (26). Briefly, SalI/PvuI-lineralized plasmid was transferred into fibroblasts by Lipofectame 2000 and screened with neomycin. Healthy transferred pIN donor cells (fibroblasts) were then cultured in starved medium and injected into the perivitelline space of enucleated oocytes with a beveled micropipette. In addition, the activated reconstructed couplets were cultured in the oviducts of synchronized temporary intermediate recipients or transferred into the uterine tubes of each recipient. After 5 days of culture in vivo of the reconstructed embryo, the developed morulae and blastocytes were surgically transferred into the uteri of synchronized final recipients. At day 35, the surrogates were scanned with B-ultrasound scanner to detect pregnant goats. Four cloned goats were eventually born.

\section{DNA extraction and genomic PCR identification}

DNA extraction. Whole goat blood genomic DNA was extracted from the four IGF-1 transgenic goats and three non-transgenic goats with the TIANamp Blood DNA Midi kit [Tiangen Biotech (Beijing) Co., Ltd, Beijing, China] according to the manufacturer's instructions. The extracted DNA was kept at $-20^{\circ} \mathrm{C}$ until use. This animal study was approved by the Institutional Animal Care and Use Committee of Nanjing Agricultural University. The extracted genomic DNA was quantified by electrophoresis. Gels were prepared with $1 \%$ agarose in TAE buffer with ethidium bromide (EtBr).

Primer design and PCR identification. Three pairs of specific primers were designed to evaluate the presence of the transgene. Primers were designed according to Fig. 1A and are listed in Table I. PCR amplification was performed with the genomic DNA template, recombinant Taq polymerase, $10 \mathrm{X}$ buffer, and primers. The conditions for PCR were: $94^{\circ} \mathrm{C}$ for $5 \mathrm{~min}, 94^{\circ} \mathrm{C}$ for $30 \mathrm{sec}, 60^{\circ} \mathrm{C}$ for $30 \mathrm{sec}$, and $72^{\circ} \mathrm{C}$ for $90 \mathrm{sec}$ for 30 cycles, with a final extension at $72^{\circ} \mathrm{C}$ for $10 \mathrm{~min}$. The PCR product was isolated and linked to plasmid pMD19-T for sequencing.

Southern blot analysis. Southern blotting hybridization was carried out with the DIG system (F. Hoffmann-La Roche AG, Basel, Switzerland) according to the manufacturer's instructions. Briefly, $30 \mu \mathrm{g}$ genomic DNA from four cloned goats
Table I. qPCR primers designed for detecting copy number.

\begin{tabular}{llc}
\hline Name & \multicolumn{1}{c}{ Sequence (5'-3') } & Size (bp) \\
\hline 1F & ACATCCTCCTCGCATCTCTTC & 804 \\
1R & CCTTCTTAGGTTTGTTATTCTTAGCC & 804 \\
2F & CATTGTTTGATCATATGCACCTC' & 1441 \\
2R & CCTTCTTAGGTTTGTTATTCTTAGCC & 1441 \\
3F & CTCTGGTTCCTCTGCCTTTTC & 1184 \\
3R & ATCTCCTGTCATCTCACCTTGC & 1184 \\
\hline
\end{tabular}

PCR, polymerase chain reaction.

and three control goats were digested with HindIII/EcoRI and separated on a $1.0 \%$ agarose gel. Plasmid pIN was also digested as a positive control. The gels were blotted onto nylon- $\mathrm{N}^{+}$ membrane (Whatman plc., Maidstone, England) overnight in 20X SSC buffer. As a probe, 804 bp amplified fragments were labeled with DIG-11-dUTP using the PCR DIG Probe Synthesis kit and hybridized to the membrane at $45^{\circ} \mathrm{C}$ overnight in DIG Easy Hyb solution (both from F. Hoffmann-La Roche AG). Detection was performed with the DIG Wash and Block Buffer Set (F. Hoffmann-La Roche AG). The DIG-labeled probe was detected with anti-digoxigenin AP Fab fragment and visualized with nitroblue tetrazolium chloride and 5-bromo-4-chloro-3-indolyl-phosphate (NBT/BCIP) solution (both from F. Hoffmann-La Roche AG).

Detection of $q P C R$. To examine the copy numbers of the IGF-I gene in transgenic goat, it was essential to construct a standard curve (7). First, a series of standard samples containing 1, 4, 16 , 64, 256 and 1,024 copies of the $I G F-1$ gene, respectively, were prepared by mixing the wild-type genome of a Saanen dairy goat with plasmid pIN. A standard curve was drawn by plotting $\Delta \mathrm{Ct}\left(\Delta \mathrm{Ct}=\mathrm{Ct}_{\mathrm{IGF}-1}-\mathrm{Ct}_{\mathrm{GAPDH}}\right)$ against the log of $I G F-1$ gene copies of corresponding standard samples. qPCR was performed with SYBR Premix Ex Taq (Takara Bio Inc., Otsu, Japan) on a 7500 real-time PCR System (Applied Biosystems, Foster City, CA, USA) as follows: $95^{\circ} \mathrm{C}$ for $10 \mathrm{sec}$, followed by 40 two-step cycles at $95^{\circ} \mathrm{C}$ for $5 \mathrm{sec}$ and at $60^{\circ} \mathrm{C}$ for $34 \mathrm{sec}$. Primers for the $I G F-1$ and $G A P D H$ genes (GAPDH was amplified concurrently as an endogenous control) are shown in Table II. A total volume of PCR $(20 \mu \mathrm{l})$, containing $2.0 \mu \mathrm{l}$ 10X PCR buffer, 0.5X SYBR-Green I deoxyribonucleoside triphosphates, $0.4 \mathrm{mmol} / 1 \mathrm{dNTP}, 1$ unit Taq DNA polymerase, $2.0 \mu \mathrm{l}$ primers and $6 \mu \mathrm{l} 20 \mathrm{X}$ diluted cDNA as the template was also utilized. The $\mathrm{Ct}$ value was calculated by the Sequence Detection System software (Applied Biosystem). The amount of target normalized to reference was calculated by $2^{-\Delta \Delta C t}$ in qPCR. For each DNA sample (four IGF-1 transgenic goats and two non-transgenic goats), both the target and reference genes were amplified independently on the same plate and in the same experimental run in triplicate. The values are presented as mean $\pm \mathrm{SEM}$. The $\mathrm{Ct}$ value was calculated by the Sequence Detection System software (Applied Biosystems).

TAIL-PCR analysis of the integration site. To analyze the integration sites, TAIL-PCR was performed employing three 
Table II. qPCR primers designed for detecting copy numbers.

\begin{tabular}{lll}
\hline Gene & \multicolumn{1}{c}{ Sense } & \multicolumn{1}{c}{ Antisense } \\
\hline$I G F-1$ & ATGCCAGTCACATCCTCCTC & CTCCAGCCTCCTCAGATCAC \\
GAPDH & GGGTGTTGTTATACTTCTCGTGGTT & GTGATGCTGGTGCTGAGTATGTG
\end{tabular}

PCR, polymerase chain reaction; IGF-1, insulin-like growth factor I.

A
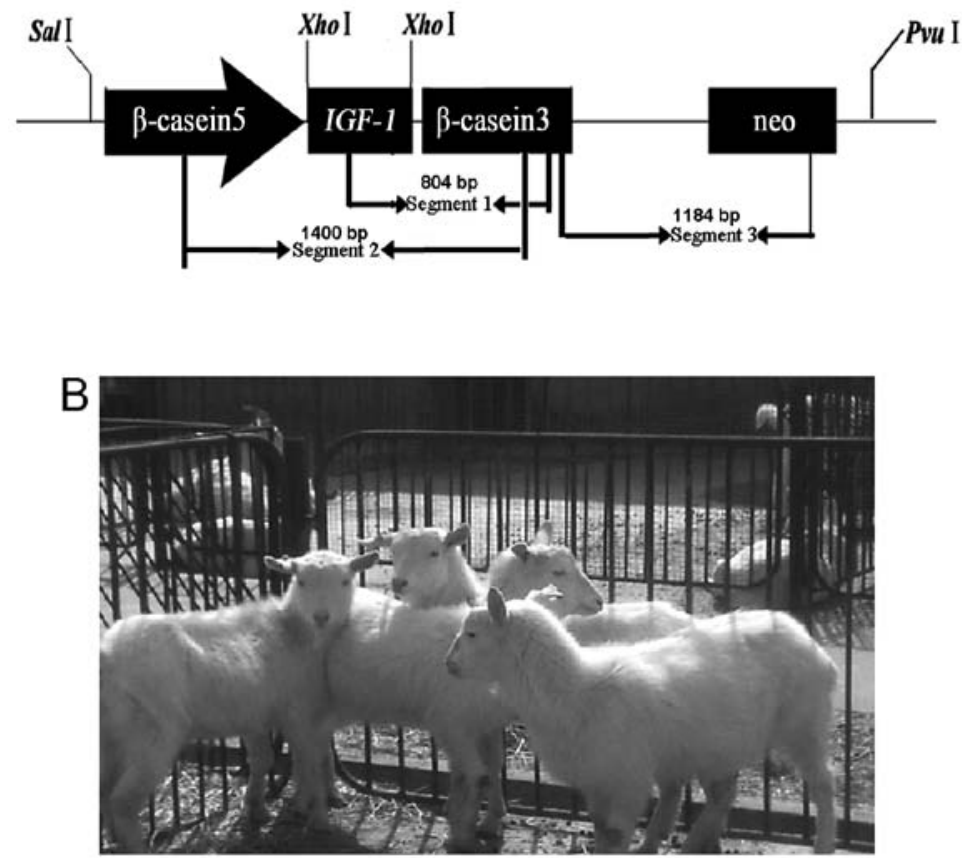

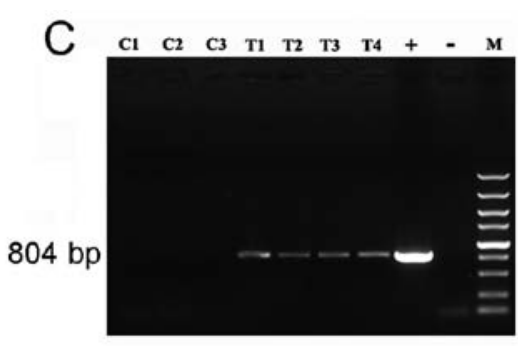

D $\quad \mathrm{Cl}^{\mathrm{C} 2} \mathrm{C}_{3} \mathrm{~T}_{1} \mathrm{~T} 2 \mathrm{~T} 2 \mathrm{~T}$ T4 $+-\mathrm{M}$
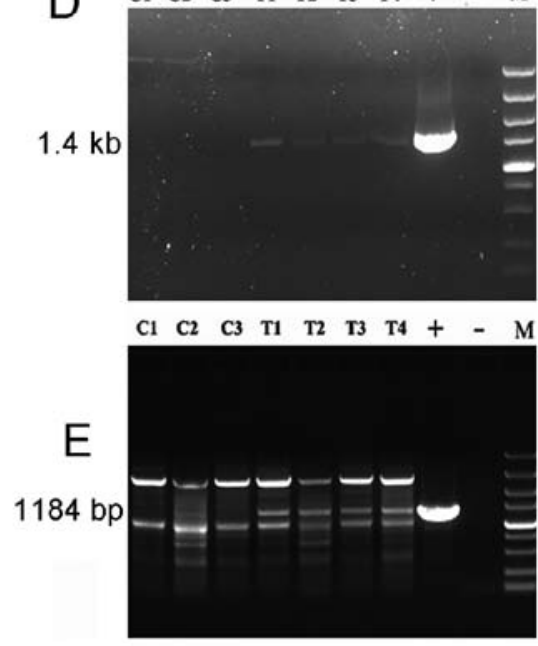

Figure 1. The production of IGF-1 transgenic goats and its identification. (A) The schematic diagram of vector pIN and primer design map ( $\beta$-casein 5 , $\beta$-casein promoter genes of goat; IGF-1, insulin-like growth factor I gene of goat; $\beta$-casein $3, \beta$-casein genomic fragment; neo, neomycin resistance gene. (B) IGF-1 transgenic goats (four healthy kids were born and survived to maturity). (C) The identification of IGF-1 from IGF-1 to 3' $\beta$-castein; (C1, C2 and C3, control goats; T1, T2, T3 and T4, IGF-1 transgenic goats; +, mammary gland-specific expression vector pIN; -, negative ; M, Marker DNA/Trans2K Plus). (D) The identification of IGF-1 from 3' $\beta$-castein to $5^{\prime} \beta$-castein; (E) the identification of neo from 3 ' $\beta$-castein to neo.

transgene-specific primers and five arbitrary degenerate primers (AD1, AD2, AD3, AD4 and AW). Fig. 2A shows the schematic relationship between the specific primers and arbitrary degenerate primers with the target genomic sequence or flanking sequence of IGF-1 transgenic insertion. All the primers used in this study were purchased from Invitrogen Co., Ltd. (Shanghai, China) and are listed in Table III. The TAIL-PCR process included three sequential PCR reactions (Table IV). TAIL-PCR protocol was performed according to the method described by Liu et al (27). Briefly, the primary PCR reaction contained recovered HindIII/EcoRI-digested template (two types of template were used; one was a fragment $<4 \mathrm{~kb}$, while the other was a fragment $>4 \mathrm{~kb}$ ), $2.5 \mathrm{mM}$ each dNTPs, $0.6 \mu \mathrm{M}$ of SP1 primer, $2 \mu \mathrm{m}$ of the AD primer, and 1 unit of Taq polymerase in $20 \mu \mathrm{l}$ reaction buffer. In the secondary or tertiary round, $1 \mu \mathrm{l}$ of 10 -fold diluted first or secondary products were used as templates and supplemented in the reaction. The thermal cycling conditions are shown in Table V. The products of tertiary TAIL-PCR reaction were separated on a $1.0 \%$ agarose gel. Specific bands for each set were purified using a gel purification kit (Omega Bio-Tek, Inc., Norcross, GA, USA) and sequenced. Sequencing results were analyzed using the BLAST and NCBI databases for bovine genomic DNA to identify the specific chromosomal integration sites.

\section{Results}

Generation of transgenic goats. In total, 50 donor goats were used in this study, with 388 in vivo matured oocytes being recovered. In addition, 375 oocytes were enucleated and produced karyoplast-cytoplast couplets using prepared donor cells. Subsequent to fusion, a total of 221 reconstructed embryos were obtained. Reconstructed pronuclear-stage embryos were transferred into 46 synchronized recipients, which produced $23(50 \%)$ pregnancies at day 35 . Four healthy kids were born and survived to maturity (Fig. 1B). 
Table III. Primers designed for TAIL-PCR.

Primer

\section{AD1}

AD2

AD3

AD4

AW

Special primers

SalI3

SalI2

SalI1

PvuI3

PvuI2

$P v u \mathrm{I} 1$
Sequence

\section{TGCACCACTGGACTGAGCGGCCGCVNVNNNGGAA TGCACCACTGGACTGAGCGGCCGCBNBNNNGGTT TGCACCACTGGACTGAGCGGCCGCVVNVNNNCCAA TGCACCACTGGACTGAGCGGCCGCVBNBNNNCGGT TGCACCACTGGACTGA}

\author{
GAGAAGCGTTCAGAGGAAAGCGATC \\ CTCAAAGAGCAGCGAGAAGCGTTC \\ CAGGCCGTTCTATGATTCTGTCATTC \\ CGCCGCATACACTATTCTCAGAATG \\ CAACTCGGTCGCCGCATACACTATTC \\ CTTTTAAAGTTCTGCTATGTGGCGCG
}

TAIL-PCR, thermal asymmetric interlaced polymerase chain reaction. Bold letters indicate the core sequences in the AD primers.

Table IV. The reaction system of TAIL-PCR.

\begin{tabular}{|c|c|c|c|}
\hline Reaction composition & First round & Second round & Third round \\
\hline 10X ExTaq buffer & 2 & 2 & 5 \\
\hline $2.5 \mathrm{Mmol} / \mathrm{l} \mathrm{dNTP}$ & 1 & 1 & 2 \\
\hline $10 \mu \mathrm{mol} / 1 \mathrm{AD}$ & 2 & 1 & 2 \\
\hline $10 \mu \mathrm{mol} / 1 \mathrm{SP}$ & 0.6 & 1 & 2 \\
\hline $\mathrm{Ex} \mathrm{Taq} /(5 \mathrm{U} / \mu \mathrm{l})$ & 0.2 & 0.2 & 0.5 \\
\hline $\mathrm{ddH}_{2} \mathrm{O}$ & Add to $20 \mu 1$ & Add to $25 \mu 1$ & Add to $50 \mu 1$ \\
\hline Template & $\begin{array}{l}\text { Genomic DNA digested } \\
\text { with HindIII/EcoRI }\end{array}$ & $\begin{array}{c}1 \mu \mathrm{l} \text { of } 10 \text {-fold } \\
\text { diluted the first products }\end{array}$ & $\begin{array}{c}1 \mu \mathrm{l} \text { of } 10 \text {-fold } \\
\text { diluted the secondary products }\end{array}$ \\
\hline
\end{tabular}

TAIL-PCR, thermal asymmetric interlaced polymerase chain reaction.

PCR detecting of transgenic goats. When four cloned goats were previously generated by SCNT, the first step was to identify whether these cloned goats were IGF-1 transgenic goat. PCR was carried out with three pairs of primers to analyze the cloned goats. Results showed that the four goats that survived to maturity were IGF-1 transgenic. Fig. $1 \mathrm{C}$ and D show that the $I G F-1$ gene was integrated into the genome of four cloned goats, while there was no target band in the non-transgenic control goats. Sequencing results showed that the inserted IGF-1 was consistent with the plasmid pIN, without any mutations. Fig. 1E shows that four clone goats contained the IGF-I gene in their genome, and included the resistance gene (neo).

Southern blotting identification of transgenic goats. After PCR detection, we verified that plasmid pIN was integrated into the genomic DNA of transgenic goats using southern blotting. Southern blotting, a highly accurate and sensitive technology, has been widely used in the identification of genetically modified products, especially when the transferred genes were highly homologous with endogenous genomic DNA. For detection of southern blotting, a probe was designed and amplified (Fig. 2A).
Fig. 3A shows a clear band on the NC member only in the four IGF-1 transgenic goat lanes and positive plasmid pIN lane. This result, consistent with the PCR results, proved that the four clone goats were IGF-1 transgenic goats.

Copy number analysis of the IGF-1 gene in transgenic goats. Since the copy number of transgenes may greatly affect the expression of the target genes, we determined the copy numbers by qPCR. The absolute quantitative standard curve was drawn by plotting $\Delta \mathrm{Ct}\left(\Delta \mathrm{Ct}=\mathrm{Ct}_{\mathrm{IGF}-1}-\mathrm{Ct}_{\mathrm{GAPDH}}\right)$ against the log of $I G F-1$ gene copies of corresponding standard samples (Table VI). The standard curve was calculated as: $\log 2 \mathrm{~N}$ (copy number) $=-1.0244 \Delta \mathrm{Ct}+5.3576\left(\mathrm{R}^{2}=0.9963\right)($ Fig. $3 \mathrm{C})$. Following construction of the standard curve, we sequentially detected the copy numbers of transgenic goats with qPCR. The reported $\mathrm{Ct}$ values were averaged for the triplicates (Table VII). Using the equation, the number of IGF-1 transgene copies of the four transgenic goats were estimated (Table VI). Results determined that four transgenic goats contained approximately the same number of copies of the $I G F-1$ gene (7.89-9 copies), while no copies were identified for the non-transgenic goats. 
Table V. The thermal cycling conditions of TAIL-PCR.

\begin{tabular}{|c|c|c|c|c|c|c|}
\hline \multirow{3}{*}{$\begin{array}{l}\text { Reaction } \\
\text { steps }\end{array}$} & \multicolumn{2}{|c|}{ First reaction } & \multicolumn{2}{|c|}{ Second reaction } & \multicolumn{2}{|c|}{ Third reaction } \\
\hline & \multicolumn{2}{|c|}{ Temperature time } & \multicolumn{2}{|c|}{ Temperature time } & \multicolumn{2}{|c|}{ Temperature time } \\
\hline & 94 & $1 \mathrm{~min}$ & 94 & $1 \mathrm{~min}$ & 94 & $1 \mathrm{~min}$ \\
\hline 2 & 98 & $1 \mathrm{~min}$ & 94 & $30 \mathrm{sec}$ & 94 & $30 \mathrm{sec}$ \\
\hline 3 & 94 & $30 \mathrm{sec}$ & 64 & $1 \mathrm{~min}$ & 64 & $1 \mathrm{~min}$ \\
\hline 4 & 68 & $1 \mathrm{~min}$ & 72 & $2 \min$ & 72 & $2 \min$ \\
\hline 5 & 72 & $2 \min$ & 94 & $30 \mathrm{sec}$ & 94 & $30 \mathrm{sec}$ \\
\hline 6 & Go to step 3 & 5 times & 64 & $1 \mathrm{~min}$ & 64 & $1 \mathrm{~min}$ \\
\hline 7 & 94 & $30 \mathrm{sec}$ & 72 & $2 \min$ & 72 & $2 \min$ \\
\hline 8 & 25 & $3 \mathrm{~min}$ & 94 & $30 \mathrm{sec}$ & 94 & $30 \mathrm{sec}$ \\
\hline 9 & 72 & $2 \min$ & 44 & $1 \mathrm{~min}$ & 44 & $1 \mathrm{~min}$ \\
\hline 10 & 94 & $30 \mathrm{sec}$ & 72 & $2 \min$ & 72 & $2 \min$ \\
\hline 11 & 64 & $1 \mathrm{~min}$ & Go to step 2 & 15 times & Go to step 2 & 15 times \\
\hline 12 & 72 & $2 \min$ & 72 & $10 \mathrm{~min}$ & 72 & $10 \min$ \\
\hline 13 & 94 & $30 \mathrm{sec}$ & & & & \\
\hline 14 & 64 & $1 \mathrm{~min}$ & & & & \\
\hline 15 & 72 & $2 \min$ & & & & \\
\hline 16 & 94 & $30 \mathrm{sec}$ & & & & \\
\hline 17 & 44 & $1 \mathrm{~min}$ & & & & \\
\hline 18 & 72 & $2 \min$ & & & & \\
\hline 19 & Go to step 10 & 15 times & & & & \\
\hline 20 & 72 & $10 \mathrm{~min}$ & & & & \\
\hline
\end{tabular}

TAIL-PCR, thermal asymmetric interlaced polymerase chain reaction.

Table VI. Standard curve conversion.

\begin{tabular}{rccc}
\hline Copy no. & Ct (IGF-1) & $\mathrm{Ct}(\mathrm{GAPDH})$ & $\Delta \mathrm{C}(\mathrm{t})$ \\
\hline 1 & 25.51 & 20.07 & 5.45 \\
4 & 23.38 & 20.09 & 3.28 \\
16 & 21.34 & 20.26 & 1.08 \\
64 & 19.4 & 20.3 & -0.9 \\
256 & 17.81 & 20.33 & -2.53 \\
1,024 & 16.19 & 20.48 & -4.29 \\
\hline
\end{tabular}

IGF-1, insulin-like growth factor I.

Integration sites analysis of IGF-1 gene in transgenic goats. Since the transgene integration site and the resulting perturbation may greatly affect the expression of the inserted gene and its neighbors, we performed TAIL-PCR with three transgene specific primers and five arbitrary degenerate primers to clone the flanking sequence. For T1 transgenic goat, two specific fragments of $\sim 1,500$ and 2,000 bp in size were amplified when using the combination of arbitrary primer AD2 and special primer SalI3 at the third TAIL-PCR, while TAIL-PCR amplified three specific fragments of $\sim 500,1,200$ and 2,000 bp with primer AD2 and special primer $P v u \mathrm{I} 3$ in T1 transgenic goat (Fig. 2B). No such specific bands were amplified in the parallel negative control experiments using non-transgenic goat genomic DNA as a template. For the T2 transgenic goat, a unique fragment of $\sim 500 \mathrm{bp}$ was amplified with the arbitrary primer AD2 and special primer SalI3 and three special fragments of $\sim 500,1,500$ and 2,000 bp were amplified with primer AD2 and special primer $P v u \mathrm{I} 3$ at the third TAIL-PCR (Fig. 2B). These amplified DNA fragments were cloned into plasmid pMD19-T and sequenced. The sequenced DNA fragments were analyzed by Bio-edit. Results show that the cloned bands from the SalI side contained a 69 bp sequence of exogenous plasmid pIN SalI border region running into a goat genomic DNA sequence. The amplified bands from the $P v u \mathrm{I}$ side were consistent with the 160-bp sequence of exogenous plasmid pIN $P v u$ I border region in contiguity with the goat genomic DNA sequence. These results suggested that the junction sequences spanning the transgenic integration sites were correctly revealed.

The BLAST results showed that two distinct sites of integration were identified on the SalI border region, one each on chromosomes 18 (GenBank ID: NM-3104464.1; range, 823,389-823,645,92\%) and 16 (GenBank ID: NM-003104439.1; range, 9,355,733-9,355,900, 88\%) (Fig. 4). For the $P v u \mathrm{I}$ border region, we identified another two distinct integration sites mapped to chromosome 11 (GenBank ID: NM-001492908.3; range, 4,167,112-4,167,267, 85\%) and 2 (GenBank ID: NM-003103851.1; range, 11,847,579-11,847,774, 91\%) (Fig. 5). 
Table VII. Copy numbers of IGF-1 in transgenic goats.

\begin{tabular}{lccccr}
\hline $\begin{array}{l}\text { Goat } \\
\text { names }\end{array}$ & $\begin{array}{c}\mathrm{Ct} \\
(\mathrm{IGF}-1)\end{array}$ & $\begin{array}{c}\mathrm{Ct} \\
(\mathrm{GAPDH})\end{array}$ & $\Delta \mathrm{C}(\mathrm{t})$ & $\begin{array}{c}\log 2 \mathrm{~N} \\
(\text { copy no. })\end{array}$ & $\begin{array}{c}\text { Copy } \\
\text { no. }\end{array}$ \\
\hline Wild-type 1 & 33.62 & 21.2 & 12.42 & -7.36 & \\
Wild-type 2 & 32.99 & 21.65 & 11.34 & -6.25 & 9.00 \\
IGF-1-1 & 23.78 & 21.64 & 2.14 & 3.17 & 8.57 \\
IGF-1-2 & 23.64 & 21.44 & 2.2 & 3.1 & 8.06 \\
IGF-1-3 & 24.46 & 22.17 & 2.29 & 2.98 & 7.89 \\
IGF-1-4 & 24.12 & 21.8 & 2.32 & & \\
\hline
\end{tabular}

IGF-1, insulin-like growth factor I.
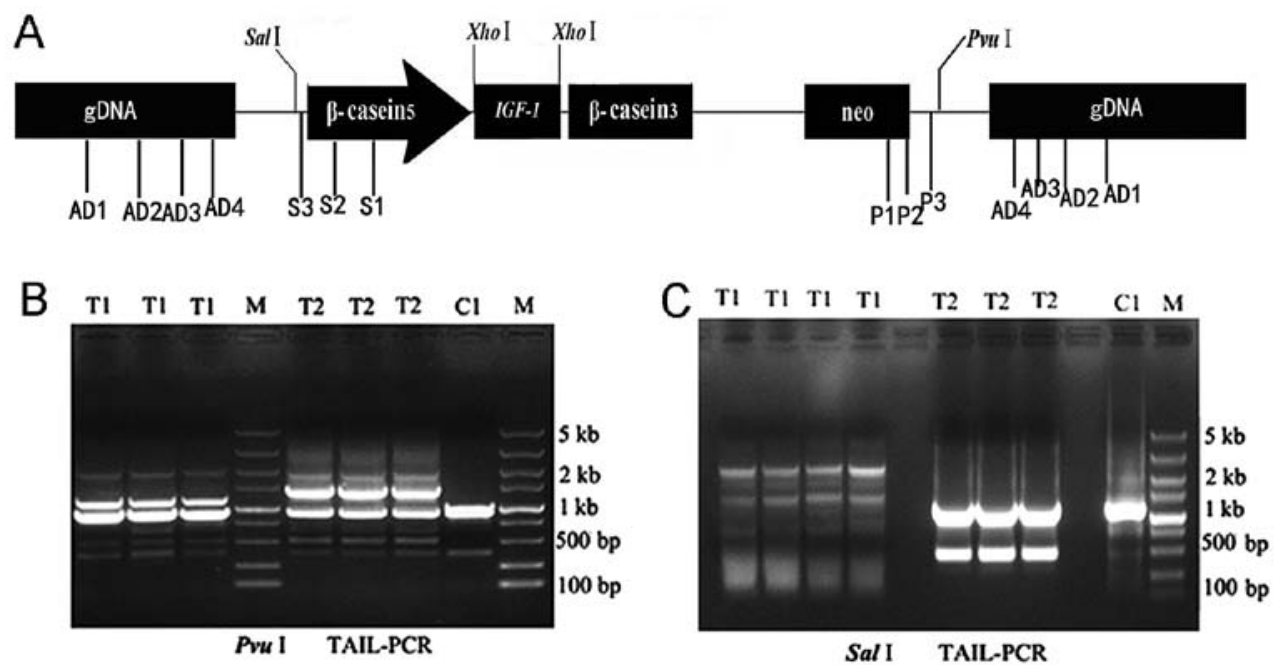

Figure 2. The design of thermal asymmetric-interlaced polymerase chain reaction (TAIL-PCR) primers and the electrophoresis of TAIL-PCR products. (A) The design map of TAIL-PCR primers [The schematic relationship between the specific primers and arbitrary degenerate primers with the target genomic sequence or flanking sequence of insulin-like growth factor I (IGF-1) transgenic insertion]. (B) The electrophoresis results of TAIL-PCR in SalI (C1, control goats; T1 and T2, IGF-1 transgenic goats; M, DL2000 marker); (C) The electrophoresis results of TAIL-PCR in PvuI; (C1, control goats; T1 and T2, IGF-1 transgenic goats; M, DL2000 marker).
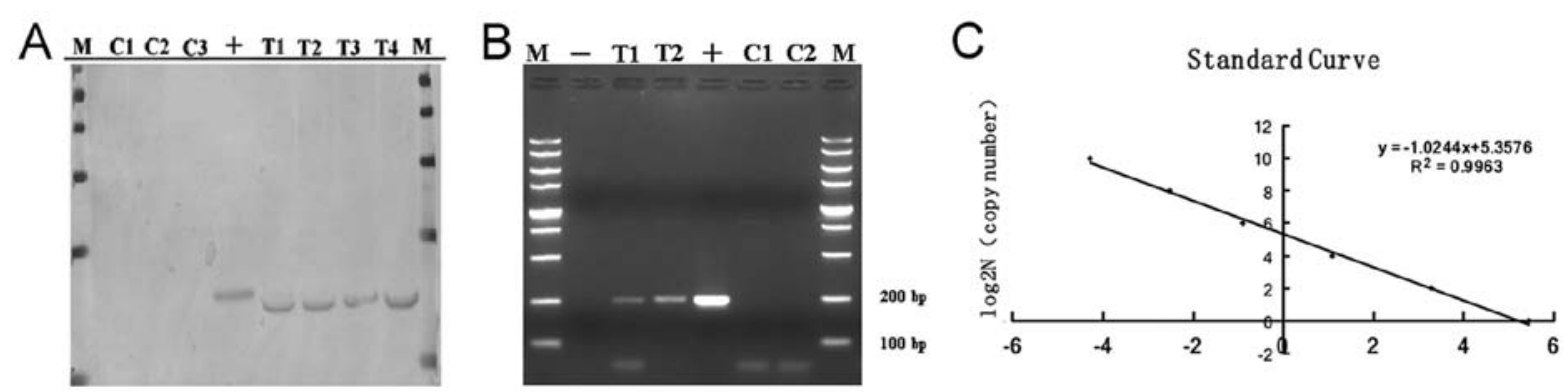

Figure 3. Southern blotting identification result of the cloned goat (genomic DNA was digested with HindIII/EcoRI, plasmid pIN was digested as positive control. A total of $804 \mathrm{bp}$ fragments from IGF-1 to 3' $\beta$-casein was amplified as a probe). (A) Southern blotting identification result of the cloned goat (lanes C1, C2 and C3, control goats; lanes T1, T2, T3 and T4, IGF-1 transgenic goats; lane +, positive control (mammary gland-specific expression vector pIN); M, DL1000 bp marker). (B) The PCR products of absolute quantitative primers (lanes C1 and C2, control goats; lanes T1 and T2, IGF-1 transgenic goats; lane +, positive control (mammary gland-specific expression vector pIN); M: DL2000 bp marker). (C) Establishment of the absolute quantitative standard curve. The standard samples containing 1,4,16,64, 256 and 1,024 copies of the IGF-1 gene were prepared. The absolute quantitative standard curve was drawn by plotting $\Delta \mathrm{Ct}\left(\Delta \mathrm{Ct}=\mathrm{Ct}_{\mathrm{IGF}-1}-\mathrm{Ct}_{\mathrm{GAPDH}}\right)$ against the log of IGF-1 gene copies of corresponding standard samples.

\section{Discussion}

The first transgenic livestock was generated almost 30 years ago (28). Currently, a common method for producing transgenic livestock involves using genetically modified cells in SCNT. Compared with conventional transgenic technology, SCNT technology allows the evaluation of the transferred gene in vitro, which may greatly improve the safety of genetically 
A

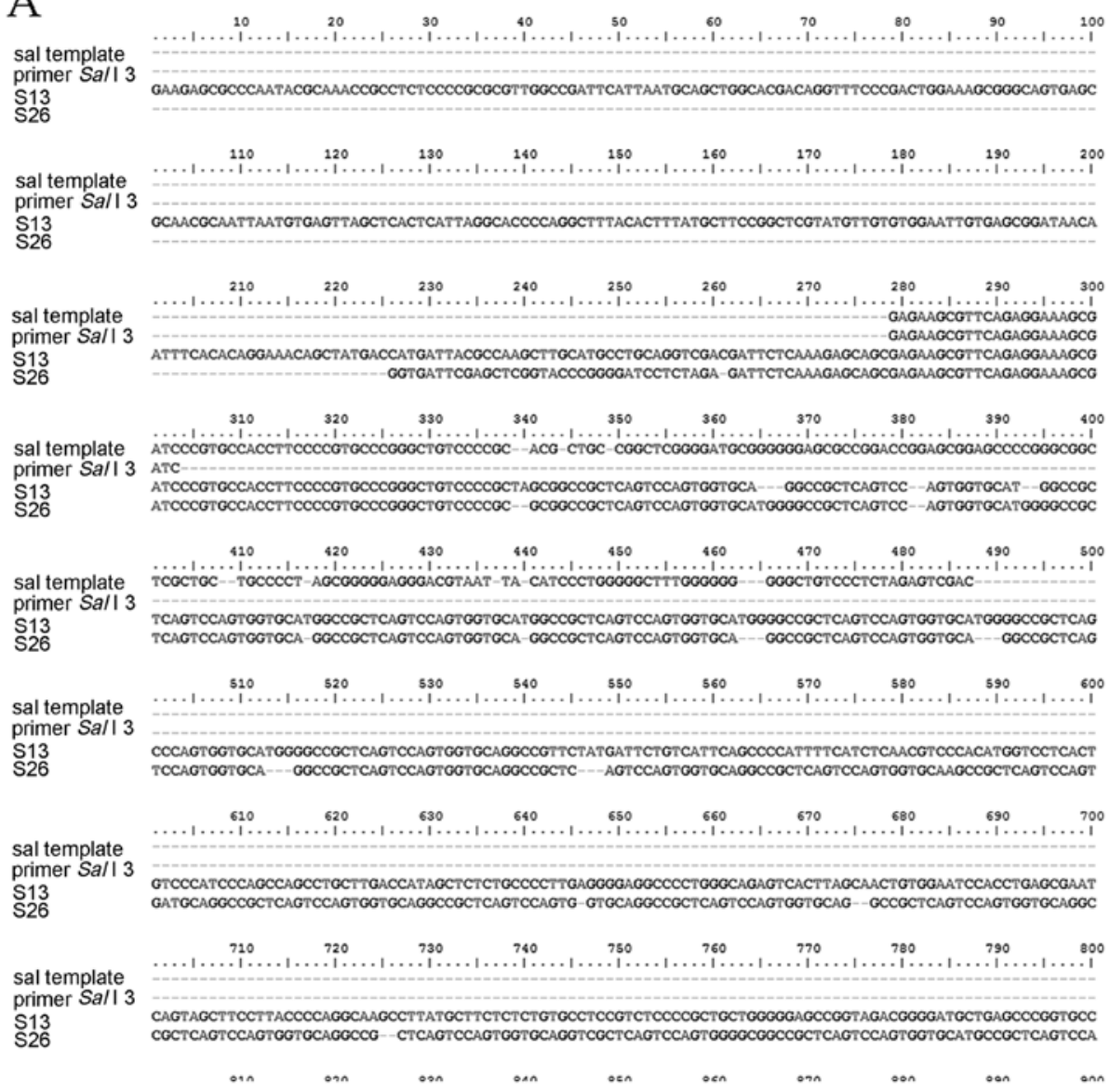

B Alignments

Bos taurus breed Hereford chromosome 18 genomic scaffold, Bos_taurus_UMD_3.1, whole genome shotgun sequence Sequence ID: reffNW_003104464.1] Length: 31414493 Number of Matches: 1 Range 1: 823389 to $8 \overline{2} 3645$

\begin{tabular}{llllll} 
Score & Expect & Identities & Gaps & Strand & Frame \\
\hline 372 bits(412) & $2 \mathrm{e}-1000$ & $237 / 257(92 \%)$ & $3 / 257(1 \%)$ & Plus/Minus &
\end{tabular}

Features: 159588 bp at 5 ' side: uncharacterized protein LOC 100847370106858 bp at 3 ' side: forkhead box protein F1

Query 66 CTGTCATTCAGCCCCATTITCATCTCAACGTCCCACATGGTCCTCACTGTCCCATCCCAG 125

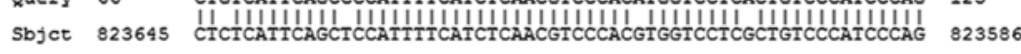

Query 126 CCAGCCTGCTTGACCATAGCTCTCTGCCCCTIGAGGGGAGGCCCCTGGGCAGAGTCACTI 185

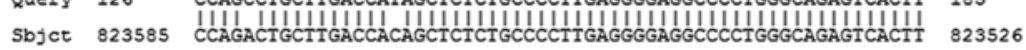

Query 186 AGCAACTGIGGAATCCACCTGAGCGAATCAGTAGCTTCCTTACCCCAGGCAAGCCTTATG 245

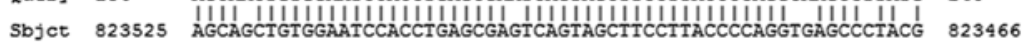

Query 246 CTTCTCTCTGTGCCTCCGTCTCCCCGCTGCTGGGGGAGCCGGTAGACG---GGGATGCTG 302

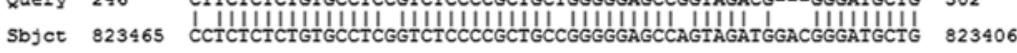

Query 303 AGCCCGGTGCCTGCCCC 319

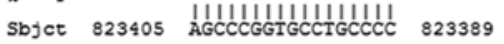

C Alignments

Bos taurus breed Hereford chromosome 16 genomic scaffold, Bos_taurus_UMD_3.1, whole genome shotgun sequence Sequence ID: reffNW_003104439.1] Length: 16624581 Number of Matches: 5 Range 1: 9355733 to 9355900

\begin{tabular}{llllll} 
Score & Expect & Identities & Gaps & Strand & Frame \\
\hline 214 bits(236) & $1 \mathrm{e}-520$ & $148 / 168(88 \%)$ & $0 / 168(0 \%)$ & Plus/Plus &
\end{tabular}

Features: 2381 bp at 5 side: PRAME family member 9/1522945 bp at 3 ' side: LOW QUALITY PROTEIN: PRAME family member 5 -like Query 413 GCAACCGGCGCACTAAGTGTGGGTGGCTGCGACCAGCACACTAAGCACGGGIGAGAGAAG 472

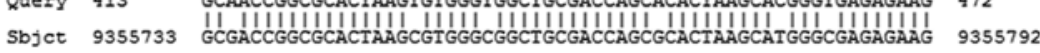

Query $473 \quad$ CTaacccacgtetgaggtcaggggcagaagetgggaggacctCATGCCTGAAGGGTGGCG 532

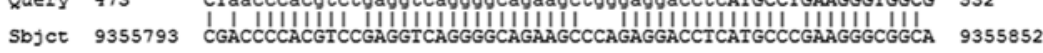

Query 533 gtcaagaggagttaccecatgtcegaggtcaggggeagtggetgggag 580

Sbjet 9355853 GCCAAGAGAGITACCCCACGTCCGAGTCAGGGAAGTGGCGAGA 9355900

Figure 4. The BLAST results of thermal asymmetric-interlaced polymerase chain reaction (TAIL-PCR) in SalI side. (A) Sequence alignment results among primer SalI3, SalI border region and TAIL-PCR product with Bio-edit software. (B) The alignment results of flank sequence from SalI side. (C) The alignment results of the flank sequence from Sall side. 
A

Pvu I template
primer Pvu I 3 P11

Pvu I template
primer Pvu I 3

primer

Pvu I template

P11

Pvu I template

P11

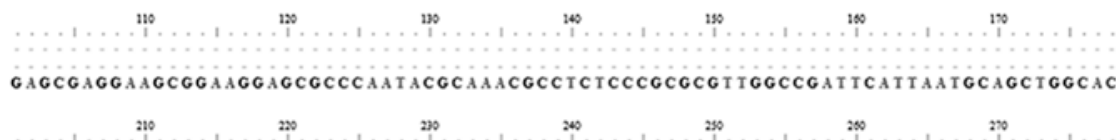

CGGGGCAGTAGCGCAACGCAATAATGGAGTAGCTCACTCATAGGCACCCCAGGCTIACACTITAGCTCCC

Pvu I template
primer Pvu I 3

P11

$\mathrm{rc}$

$$
\text { ic }
$$

Pvu I template
primer Pvu I 3 P11

AGTCTATTAATTETGCCGGGAGCTAGAGTAAGAGTCGCCAGTAATAGTTGCGCAACGTGTTGCCATTCCA

Pvu I template
primer Pvu I 3

P11

Pvu I template
primer Pvu I 3

Primer

GTGTIGGATGGCTCATCAGCTCCGGTCCCAACGACAAGGGAGTACATGATCCCCCATGTGTGCAAAAA

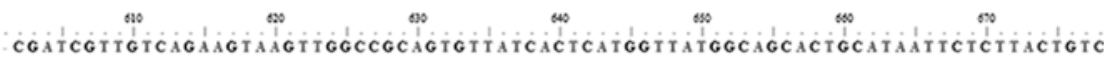
CCGATCGTTGTCAGATAATTGGCCGAGTGTATCACTCATGGTATGGCAGCACTGCATAATCTCTTACTGTC

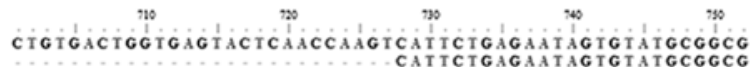

CIGTGACTGGTGAGACTCAACCAAGTCATCTGAGAATAGTGIAGCGGCGAATCICTAGAGGATCCCCGGGACCG

\section{B Alignments}

Bos taurus breed Hereford unplaced genomic scaffold, alternate assembly Btau_4.6.1 ChrUn.004-5.11787.scaffold1 Sequence ID: reffNW_001501683.1| Length: 1014 Number of Matches: 1 Range 1: 656 to 962

\begin{tabular}{|c|c|c|c|c|c|c|}
\hline Score & & Expect & Identities & Gaps & Strand & \\
\hline 219 bit: & 242) & $3 e-540$ & $237 / 309(77 \%)$ & $5 / 309(1 \%)$ & Plus/Plus & \\
\hline Feature & & & & & & \\
\hline Query & 295 & CCGCTCACAF & & & GTGTAAAGCCTGGGGIGCC & 354 \\
\hline Sbjet & 656 & CCGCCCACA & CCALACANACA & CGGAAGCA & GCGCAAAGCCGGGGGGGCC & 715 \\
\hline Query & 355 & TAATGAGTGP & CTAACTCACATI & CGITGCGCTC & GCCCCGCTTTCCAGTCGGG & 414 \\
\hline Sbjet & 716 & & & AGC & GCCCCGCCCACCAGGCGGG & 774 \\
\hline Query & 415 & AAACCTGTCC & GCCAGCTCCATT & ATCGGCCAAC & C-GGGAGAGGC-GTTTGCG & 472 \\
\hline Sbjet & 775 & & & & & 834 \\
\hline Query & 473 & TATTGGGCG & 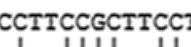 & AACTGACTC & CGCTCGGICGITCGGCTGC & 532 \\
\hline Sbjet & 835 & AAAAGGGCGC & & & CGCACGGACGNACGGCCGC & 893 \\
\hline Query & 533 & & CICACTC & GTAATACC & CCACAGAATCAGGGGATA & 591 \\
\hline Sbjet & 894 & & & & NCCACAGAACCAGGGGAAA & 953 \\
\hline Query & 592 & & 600 & & & \\
\hline Sbjet & 954 & ACGCAGGAA & 962 & & & \\
\hline
\end{tabular}

Bos taurus breed Hereford chromosome 11 genomic scaffold, alternate assembly Btau_4.6.1 Chr11.scaffold8 Sequence ID: reffNW_001492908.3| Length: 5730378 Number of Matches: 2

Range 1: 4167112 to $\overline{4} 167267$

\begin{tabular}{|c|c|c|c|c|c|c|}
\hline \multicolumn{2}{|l|}{ Score } & Expect & Identities & Gaps & Strand & \\
\hline \multicolumn{2}{|c|}{176 bits(194) } & $3 e-410$ & $134 / 157(85 \%)$ & $1 / 157(0 \%)$ & Plus/Plus & \\
\hline \multicolumn{7}{|c|}{$\begin{array}{l}\text { Features: } \\
147431 \text { bp at } 5 \text { ' side: } 3-0 \times 0-5 \text {-alpha-steroid } 4 \text {-dehydrogenase } 28024 \text { bp at 3' side: protein MEMO1 }\end{array}$} \\
\hline $\begin{array}{l}\text { Query } \\
\text { Sbjct }\end{array}$ & 4167112 & \multicolumn{4}{|c|}{ 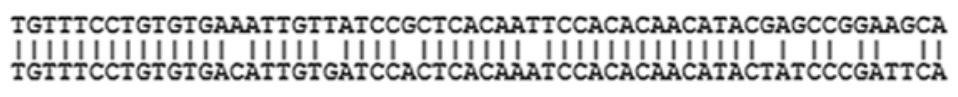 } & $\begin{array}{l}331 \\
4167171\end{array}$ \\
\hline $\begin{array}{l}\text { Query } \\
\text { Sbjct }\end{array}$ & 4167172 & \multicolumn{4}{|c|}{ 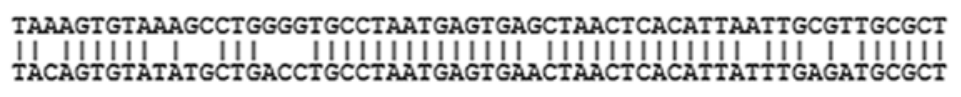 } & $\begin{array}{l}391 \\
4167231\end{array}$ \\
\hline $\begin{array}{l}\text { Query } \\
\text { Sbjct }\end{array}$ & 4167232 & \multicolumn{4}{|c|}{ 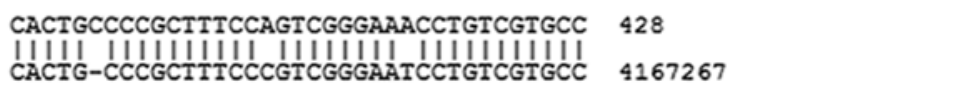 } & \\
\hline
\end{tabular}

Figure 5. The blast results of thermal asymmetric-interlaced polymerase chain reaction (TAIL-PCR) in the $P v u I$ side. (A) Sequence alignment results among primer $P v u \mathrm{I} 3, P v u \mathrm{I}$ border region and TAIL-PCR product with Bio-edit software. (B) The alignment results of the flank sequence from the $P v u \mathrm{I}$ side. 
C

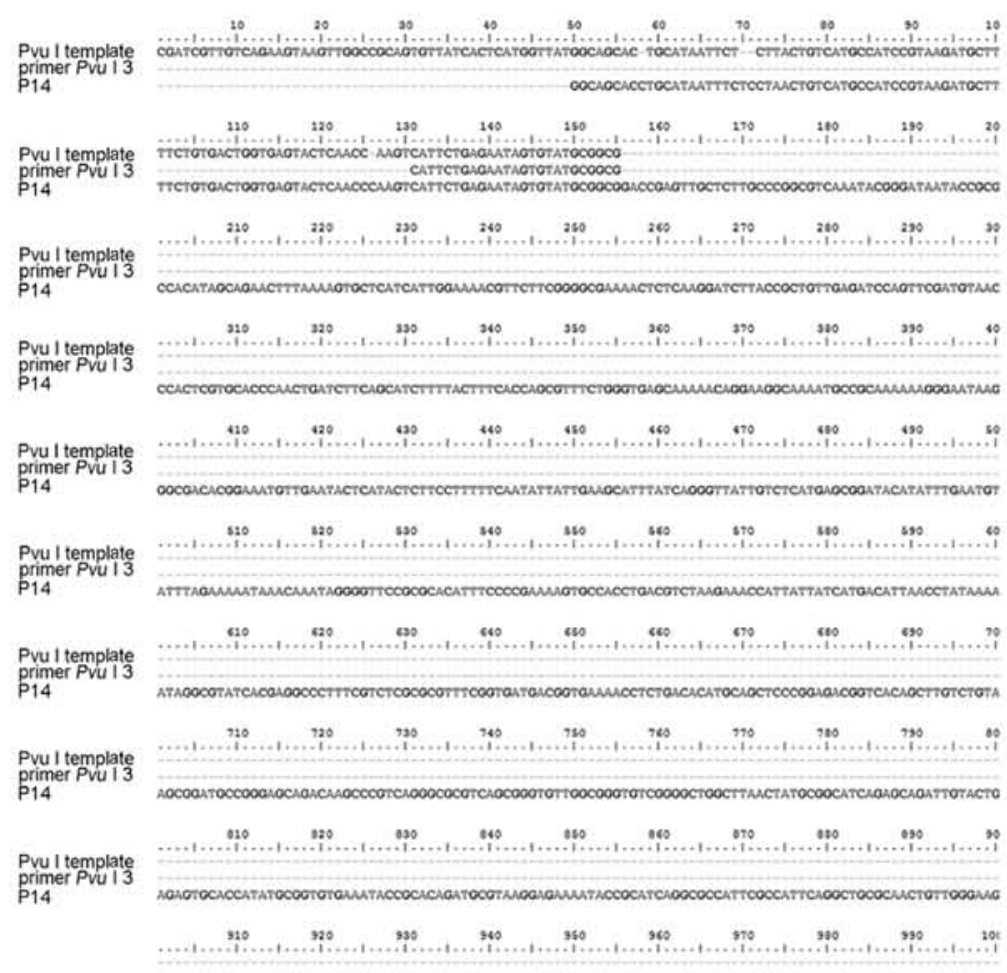

\section{Alignments}

Bos taurus breed Hereford unplaced genomic scaffold, alternate assembly Btau_4.6.1 ChrUn.004-5.11787. scaffold1 Sequence ID: refinW_001501683.1] Length: 1014 Number of Matches: 1 Range 1: 656 to 1014

\begin{tabular}{|c|c|c|c|c|c|c|}
\hline Score & & Expect & Identities & Gaps & Strand & \\
\hline 345 bits(? & (382) & $4 e-920$ & $291 / 359(81 \%)$ & $0 / 359(0 \%)$ & Plus/Plus & \\
\hline Features: & & & & & & \\
\hline Query & 68 & & & & AAGTGTAAAGCCTGGGGTGCC & 127 \\
\hline Sbjet & 656 & CCGCCCAC & $C A C A C A A C A$ & EGGAAGC: & GCGCAAAGCCGGGGGGGCC & 715 \\
\hline Query & 128 & & & & CTGCCCGCTTICCAGTCGGGA & 187 \\
\hline Sbjet & 716 & CAAAGAGCC & CNAACACACAA & CGCAGCGC & GCCCGCCCACCAGGCGGGA & 775 \\
\hline Query & 188 & AACCTGTCG & CCCAGCTGCATTA & TCGGCCARC & GCGGGGAGAGGGGGTITGCGT & 247 \\
\hline Sbict & 776 & AACCAGGC & & ACGGCCA & GGGGAGAGGCGGCCCCGCGA & 835 \\
\hline Query & 248 & & & & GTCGITCGGCTGCGG & 307 \\
\hline Sbjet & 836 & & & & CGCACGGACGNACGGCCGCGG & 895 \\
\hline Query & 308 & & & & ICCACAGAATCAGGGGATAAC & 367 \\
\hline Sbjet & 896 & & & & NCCACAGAACCAGGGGAAAAC & 955 \\
\hline Query & 368 & & CATGTGAGCA & AGCAAAAG & GGAACCGTAAAAAGGCCGC & 426 \\
\hline Sbjet & 956 & & & & GGAACCGAAAAAAGGCCGC & 1014 \\
\hline
\end{tabular}

Bos taurus breed Hereford chromosome 2 genomic scaffold, Bos_taurus_UMD_3.1, whole genome shotgun sequence Sequence ID: refiNW_003103851.1| Length: 12151094 Number of Matches: 2 Range 1: 11847579 to 11847774

\begin{tabular}{|c|c|c|c|c|c|}
\hline \multicolumn{2}{|l|}{ Score } & Expect & Identities & Frame & \\
\hline \multicolumn{2}{|c|}{260 bits(288) } & $1 e-660$ & $180 / 197(91 \%)$ & $5 / 197(2 \%)$ & \\
\hline \multicolumn{6}{|c|}{$\begin{array}{l}\text { Features: } \\
54450 \text { bp at } 5^{\circ} \text { side: ephrin type-A receptor } 2 \text { precursor } 3028 \text { bp at } 3^{\prime} \text { side: protein FAM131C }\end{array}$} \\
\hline Query & 21847579 & \multicolumn{3}{|c|}{ 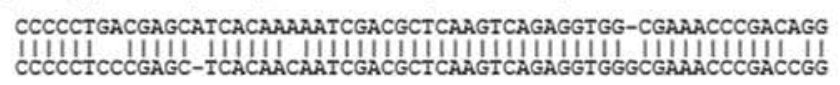 } & 11847637 \\
\hline Query & 11847638 & \multicolumn{3}{|c|}{ 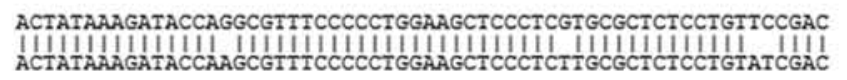 } & 11847697 \\
\hline Query & 11847698 & \multicolumn{3}{|c|}{ 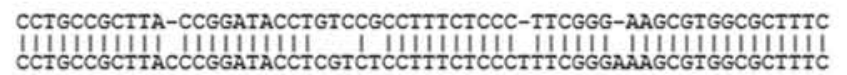 } & 11847757 \\
\hline Query & 11847758 & \multicolumn{3}{|c|}{$\begin{array}{l}\text { ICATAGCTCACGCTGIA } \\
\text { IIIIIIIIIIII11 } \\
\text { TCATAGCTCACGCTGIA }\end{array}$} & \\
\hline
\end{tabular}

Figure 5. Continued. (C) Sequence alignment results among primer $P v u \mathrm{I} 3, P v u \mathrm{I}$ border region and TAIL-PCR product with Bio-edit software. (D) The alignment results of the flank sequence from the $P v u I$ side. 
modified animal by assessing positive donor cells, and markedly reduce the probability of the transgene being silenced in offspring (29-31). In this study, we obtained four IGF-1 transgenic goats, which were developed to adolescence and expected to be bred in September 2013. The four goats have suitably developed in body and mammary gland, without any disease.

To evaluate the transgenically cloned goat for commercial use, the transgene copies and integration sites should be identified. The transgene in the present study was a 465 bp IGF-1 fragment in the $\mathrm{pBC} 1$ vector. $\mathrm{PCR}$ and southern blotting results demonstrated that all four goats were IGF-1 transgenic goats. It is known that the transgene may randomly integrate into any site of the genome, the integration site and copies may be influenced by the insert time, plasmid form and insert position (32). Both the number of copies are presently known to integrate and the genomic context of the transgene has been proven to influence the phenotype of the transgenic animal $(7,33,34)$. The high copy number tandem integration was thought to lead to transgene silencing $(35,36)$ and the high copy number may decrease with aging in transgenic animals. Ballester et al and Vaisman detected the copies of transgenic animal using qPCR methods $(11,37)$. The study by Kong et al suggested that the transgene expression level is associated with the copy number in transgenic pigs (7). In this study, we aimed to identify the copy numbers in IGF-1 transgenic goats with qPCR. qPCR is considered a simple, rapid and accurate method to estimate the transgene copy number in transgenic animals. By using this method, we revealed that four transgenic goats contained almost the same copy numbers of the transferred IGF-1 gene in goat genome (from 7.89 to 9 copies). For a similar copy number of the four transgenic goats, the same donor cell may be an appropriate explanation.

Following evaluation of the copy numbers of IGF-1 transgenic goats, we detected the integration sites of the transgenic goats, which primarily affect the transgene expression. The integration site has been proven to affect the level and time of transgene expression $(38,39)$. In transgenic mouse, the same transferred gene would be transcribed in different time periods for their different insert location (40). However, results of recent studies have suggested that the integration site may not always be random (42). Previously it was found that in certain sites the transgene was inclined to integrate by random transfection, such as LINE elements (41). However, random transfection may not result in random integration but in some hot integration sites and these hot integration sites may have a common character allowing the transgene to integrate. Yan et al demonstrated that the foreign fad 2 behaved similar to an X-linked gene and that foreign DNA molecules were inserted into the eukaryotic genome through a homologous illegitimate random integration (42).

Considering the importance of identifying the integration site, several PCR-based methods have been applied for the precise determination of the integration site of foreign DNA into native chromosomes. These methods include I-PCR, interspersed repetitive sequence PCR (IRS-PCR), linear amplification-mediated PCR (LAM-PCR) and TAIL-PCR. Of these, TAIL-PCR has obvious advantages as it may be used to identify homozygous animals $(14,15)$. TAIL-PCR has been widely used for genome walking and flanking sequence cloning in transgenic animals. In TAIL-PCR, enrichment of the target products depends on the difference in amplification velocity between the target and non-target products $(13,24)$. In this study, we improved the TAIL-PCR methods. The performance of TAIL-PCR was strongly dependent on the PCR efficiency and specificity. The improved methods entailed one more digestion reaction. When we performed the TAIL-PCR, we first digested the template goat genome DNA by HindIII and EcoRI, which was used in southern blotting identification. The digested genome was run on $0.8 \%$ agarose gel and then recovered; the fragments were $>4$ or $<4 \mathrm{~kb}$. TAIL-PCR with subsequently performed using the two types of templates as described in a previous section. The digested step was used to increase the specificity of target genes. Compared with conventional TAIL-PCR, our methods divided possible integration site into two sections, increasing the efficiency of TAIL-PCR, particularly for the template $<4 \mathrm{~kb}$. The results suggest that the improved TAIL-PCR was more efficient than conventional TAIL-PCR for cloning flanking sequences. Using these modified methods, we identified the integration sites NM-001492908.3, NM-003103851.1, NM-003104439.1, NM-3104464.1.

In conclusion, PCR-based techniques have been widely used for the precise transgene flanking sequence and copy number identification in molecular biology research. The present study has demonstrated the successful use of PCR and southern blotting to characterize four cloned goats as IGF-1 transgenic goats. The qPCR method clarified the copy numbers of the $I G F-1$ gene in transgenic goats. Furthermore, using the TAIL-PCR approach, we identified four integration sites with high specificity and provided information on the chromosomal location. The future application of TAIL-PCR to characterize transgenic animals is likely to be extremely significant.

\section{Acknowledgements}

This study was supported by the National Animal Transgenic Breeding Grand Project of China (no. 2013ZX08008-004) and a project funded by the Priority Academic Program Development of Jiangsu Higher Education Institutions.

\section{References}

1. Houdebine LM: The methods to generate transgenic animals and to control transgene expression. J Biotechnol 98: 145-160, 2002.

2. Maksimenko OG, Deykin AV, Khodarovich YM and Georgiev PG: Use of transgenic animals in biotechnology: prospects and problems. Acta Naturae 5: 33-46, 2013.

3. Ménoret S, Tesson L, Rémy $\mathrm{S}$, et al: Technical advances in the generation of transgenic animals and in their applications. Transgenic Res 22: 1065-1072, 2013.

4. Niemann H, Kues W and Carnwath JW: Transgenic farm animals: present and future. Rev Sci Tech 24: 285-298, 2005.

5. Zhang R, Yin Y, Zhang Y, et al: Molecular characterization of transgene integration by next-generation sequencing in transgenic cattle. PLoS One 7: e50348, 2012.

6. Chandler KJ, Chandler RL, Broeckelmann EM, Hou Y Southard-Smith EM and Mortlock DP: Relevance of BAC transgene copy number in mice: transgene copy number variation across multiple transgenic lines and correlations with transgene integrity and expression. Mamm Genome 18: 693-708, 2007.

7. Kong Q, Wu M, Huan Y, et al: Transgene expression is associated with copy number and cytomegalovirus promoter methylation in transgenic pigs. PLoS One 4: e6679, 2009. 
8. Pena RN, Webster J, Kwan S, Korbel J and Whitelaw BA: Transgene methylation in mice reflects copy number but not expression level. Mol Biotechnol 26: 215-220, 2004.

9. Garrick D, Fiering S, Martin DI and Whitelaw E: Repeat-induced gene silencing in mammals. Nat Genet 18: 56-59, 1998.

10. Hogan B: Molecular biology. Enhancers, chromosome position effects, and transgenic mice. Nature 306: 313-314, 1983.

11. Ballester M, Castelló A, Ibáñez E, Sánchez A and Folch JM: Real-time quantitative PCR-based system for determining transgene copy number in transgenic animals. Biotechniques 37 : 610-613, 2004

12. Mitrecić D, Huzak M, Curlin M and Gajović S: An improved method for determination of gene copy numbers in transgenic mice by serial dilution curves obtained by real-time quantitative PCR assay. J Biochem Biophys Methods 64: 83-98, 2005.

13. Huang H, Wang G, Zhao Y, Shi P, Luo H and Yao B: Direct and efficient cloning of full-length genes from environmental DNA by RT-qPCR and modified TAIL-PCR. Appl Microbiol Biotechnol 87: 1141-1149, 2010.

14. Singer T and Burke E: High-throughput TAIL-PCR as a tool to identify DNA flanking insertions. Methods Mol Biol 236: 241-272, 2003.

15. Thanh T, Chi VT, Abdullah MP, Omar H and Napis S: Efficiency of ligation-mediated PCR and TAIL-PCR methods for isolation of $\mathrm{RbcS}$ promoter sequences from green microalgae Ankistrodesmus convolutus. Mol Biol (Mosk) 46: 64-70, 2012.

16. Mason G, Provero P, Vaira AM and Accotto GP: Estimating the number of integrations in transformed plants by quantitative real-time PCR. BMC Biotechnol 2: 20, 2002.

17. Alexander GM, Erwin KL, Byers N, et al: Effect of transgene copy number on survival in the G93A SOD1 transgenic mouse model of ALS. Brain Res Mol Brain Res 130: 7-15, 2004.

18. Ganss R, Montoliu L, Monaghan AP and Schutz G: A cell-specific enhancer far upstream of the mouse tyrosinase gene confers high level and copy number-related expression in transgenic mice. EMBO J 13: 3083-3093, 1994

19. Zimonjic DB,Ullmannova-Benson V,Factor VM, Thorgeirsson SS and Popescu NC: Recurrent and nonrandom DNA copy number and chromosome alterations in Myc transgenic mouse model for hepatocellular carcinogenesis: implications for human disease. Cancer Genet Cytogenet 191: 17-26, 2009.

20. Pillai MM, Venkataraman GM, Kosak S and Torok-Storb B: Integration site analysis in transgenic mice by thermal asymmetric interlaced (TAIL)-PCR: segregating multiple-integrant founder lines and determining zygosity. Transgenic Res 17: 749-754, 2008.

21. Triglia T, Peterson MG and Kemp DJ: A procedure for in vitro amplification of DNA segments that lie outside the boundaries of known sequences. Nucleic Acids Res 16: 8186, 1988.

22. Yuanxin Y, Chengcai A, Li L, Jiayu G, Guihong $T$ and Zhangliang C: T-linker-specific ligation PCR (T-linker PCR): an advanced PCR technique for chromosome walking or for isolation of tagged DNA ends. Nucleic Acids Res 31: e68, 2003.

23. Liu YG and Whittier RF: Thermal asymmetric interlaced PCR: automatable amplification and sequencing of insert end fragments from P1 and YAC clones for chromosome walking. Genomics 25: 674-681, 1995

24. Tonooka Y and Fujishima M: Comparison and critical evaluation of PCR-mediated methods to walk along the sequence of genomic DNA. Appl Microbiol Biotechnol 85: 37-43, 2009.

25. Lin J, Yu Q, Zhang Q and Yang Q: Construction of mammary gland specific expression plasmid pIN and its expression in vitro and in vivo. Afr J Biotechnol 11: 6946-6955, 2012.
26. Zou X, Wang Y, Cheng Y, et al: Generation of cloned goats (Capra hircus) from transfected foetal fibroblast cells, the effect of donor cell cycle. Mol Reprod Dev 61: 164-172, 2002.

27. Liu YG, Mitsukawa N, Oosumi T and Whittier RF: Efficient isolation and mapping of Arabidopsis thaliana T-DNA insert junctions by thermal asymmetric interlaced PCR. Plant J 8: 457-463, 1995.

28. Hammer RE, Pursel VG, Rexroad CE Jr., et al: Production of transgenic rabbits, sheep and pigs by microinjection. Nature 315 : 680-683, 1985.

29. Goissis MD, Suhr ST and Cibelli JB: Effects of donor fibroblasts expressing OCT4 on bovine embryos generated by somatic cell nuclear transfer. Cell Reprogram 15: 24-34, 2013.

30. Niemann H and Lucas-Hahn A: Somatic cell nuclear transfer cloning: practical applications and current legislation. Reprod Domest Anim 47 (Suppl 5): 2-10, 2012.

31. Zhao MT, Lin H, Liu FJ, et al: Efficiency of human lactoferrin transgenic donor cell preparation for SCNT. Theriogenology 71: 376-384, 2009.

32. Eszterhas SK, Bouhassira EE, Martin DI and Fiering S: Transcriptional interference by independently regulated genes occurs in any relative arrangement of the genes and is influenced by chromosomal integration position. Mol Cell Biol 22: 469-479, 2002.

33. Bender B, Bodrogi L, Mayer B, et al: Position independent and copy-number-related expression of the bovine neonatal $\mathrm{Fc}$ receptor alpha-chain in transgenic mice carrying a $102 \mathrm{~kb}$ BAC genomic fragment. Transgenic Res 16: 613-627, 2007.

34. Rahman MA, Hwang GL, Razak SA, Sohm F and Maclean N: Copy number related transgene expression and mosaic somatic expression in hemizygous and homozygous transgenic tilapia (Oreochromis niloticus). Transgenic Res 9: 417-427, 2000.

35. Smith CJ, Nedwell DB, Dong LF and Osborn AM: Evaluation of quantitative polymerase chain reaction-based approaches for determining gene copy and gene transcript numbers in environmental samples. Environ Microbiol 8: 804-815, 2006.

36. Tang W, Newton RJ and Weidner DA: Genetic transformation and gene silencing mediated by multiple copies of a transgene in eastern white pine. J Exp Bot 58: 545-554, 2007.

37. Vaisman BL: Genotyping of transgenic animals by real-time quantitative PCR with TaqMan probes. Methods Mol Biol 1027: 233-251, 2013.

38. Bressan FF, Dos Santos Miranda M, Perecin F, et al: Improved production of genetically modified fetuses with homogeneous transgene expression after transgene integration site analysis and recloning in cattle. Cell Reprogram 13: 29-36, 2011.

39. Chawla R, Ariza-Nieto M, Wilson AJ, Moore SK and Srivastava V: Transgene expression produced by biolistic-mediated, site-specific gene integration is consistently inherited by the subsequent generations. Plant Biotechnol J 4: 209-218, 2006.

40. Woychik RP and Alagramam K: Insertional mutagenesis in transgenic mice generated by the pronuclear microinjection procedure. Int J Dev Biol 42: 1009-1017, 1998.

41. Migliaccio AR, Bengra C, Ling J, et al: Stable and unstable transgene integration sites in the human genome: extinction of the green fluorescent protein transgene in K562 cells. Gene 256: 197-214, 2000.

42. Yan B, Li D and Gou K: Homologous illegitimate random integration of foreign DNA into the $\mathrm{X}$ chromosome of a transgenic mouse line. BMC Mol Biol 11: 58, 2010. 